\title{
Froissart bound on inelastic cross section without unknown constants
}

\author{
André Martin ${ }^{1, *}$ and S. M. Roy ${ }^{2, \dagger}$ \\ ${ }^{1}$ Theoretical Physics Division, CERN, 1211 Geneva 23, Switzerland \\ ${ }^{2}$ HBCSE, Tata Institute of Fundamental Research, VN Purav Marav Marg, Mumbai 400088, India
}

(Received 9 March 2015; published 17 April 2015)

\begin{abstract}
Assuming that axiomatic local field theory results hold for hadron scattering, André Martin and S. M. Roy recently obtained absolute bounds on the $D$ wave below threshold for pion-pion scattering and thereby determined the scale of the logarithm in the Froissart bound on total cross sections in terms of pion mass only. Previously, Martin proved a rigorous upper bound on the inelastic cross-section $\sigma_{\text {inel }}$ which is onefourth of the corresponding upper bound on $\sigma_{\mathrm{tot}}$, and $\mathrm{Wu}$, Martin, Roy and Singh improved the bound by adding the constraint of a given $\sigma_{\mathrm{tot}}$. Here we use unitarity and analyticity to determine, without any highenergy approximation, upper bounds on energy-averaged inelastic cross sections in terms of lowenergy data in the crossed channel. These are Froissart-type bounds without any unknown coefficient or unknown scale factors and can be tested experimentally. Alternatively, their asymptotic forms, together with the Martin-Roy absolute bounds on pion-pion $D$ waves below threshold, yield absolute bounds on energy-averaged inelastic cross sections. For example, for $\pi^{0} \pi^{0}$ scattering, defining

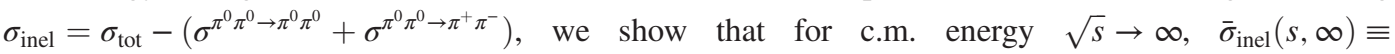
$s \int_{s}^{\infty} d s^{\prime} \sigma_{\text {inel }}\left(s^{\prime}\right) / s^{\prime 2} \leq(\pi / 4)\left(m_{\pi}\right)^{-2}\left[\ln \left(s / s_{1}\right)+(1 / 2) \ln \ln \left(s / s_{1}\right)+1\right]^{2}$ where $1 / s_{1}=34 \pi \sqrt{2 \pi} m_{\pi}^{-2}$. This bound is asymptotically one-fourth of the corresponding Martin-Roy bound on the total cross section, and the scale factor $s_{1}$ is one-fourth of the scale factor in the total cross section bound. The average over the interval $(\mathrm{s}, 2 \mathrm{~s})$ of the inelastic $\pi^{0} \pi^{0}$ cross section has a bound of the same form with $1 / s_{1}$ replaced by $1 / s_{2}=2 / s_{1}$.

DOI: $10.1103 /$ PhysRevD.91.076006

PACS numbers: 03.67.-a, 03.65.Ud, 42.50.-p
\end{abstract}

\section{INTRODUCTION}

Recently we [1] have obtained bounds on energy averages of the total cross section without any unknown constants such as an overall constant factor or the scale factor in the logarithm. The purpose of the present work is to obtain analogous bounds on the energy-averaged inelastic cross section without any unknown constants. The background and the basic postulates are again summarized below to make this work self-contained.

The Froissart [2] bound on the total cross section $\sigma_{\text {tot }}(s)$ for two particles at c.m. energy $\sqrt{s}$,

$$
\sigma_{\text {tot }}(s) \leq_{s \rightarrow \infty} C\left[\ln \left(s / s_{0}\right)\right]^{2},
$$

(where $C, s_{0}$ are unknown constants) was initially proved assuming the Mandelstam representation. This assumption might not be valid, for example, if there are rising Regge trajectories. Fortunately, [3] it was possible to prove this bound rigorously in the much more general frame work of

\footnotetext{
*martina@mail.cern.ch

${ }^{\dagger}$ smroy@hbcse.tifr.res.in
}

Published by the American Physical Society under the terms of the Creative Commons Attribution 3.0 License. Further distribution of this work must maintain attribution to the author $(s)$ and the published article's title, journal citation, and DOI.
Wightman's [4] axiomatic local field theory as applied to hadrons. Later, the needed analyticity properties, and polynomial boundedness at fixed momentum transfer squared $t$, were obtained by Epstein, Glaser and Martin [5] in the even more general framework of the theory of local observables of Haag, Kastler and Ruelle [6]. It has nevertheless been questioned [7] if these properties apply to hadrons made of quarks and gluons. Zimmermann [8] has shown that local fields can be associated to composite particles. We decide to believe that this proof applies to the present situation. We postulate that the analyticity and polynomial boundedness derived from local field theory holds for hadrons.

In the proof of the Froissart bound in [3] a crucial role is played by the use of unitarity to enlarge the Lehmann ellipse of analyticity [9] for the absorptive part $A(s, t)$ to show that the right extremity $t_{0}$ of the enlarged ellipse in the $t$-plane stays nonzero and positive when $s \rightarrow \infty$. For many processes, for example for $\pi \pi, K K, K \bar{K}, \pi K, \pi N, \pi \Lambda$ scattering it is known [10] that $t_{0}=4 m_{\pi}^{2}, m_{\pi}$ being the pion-mass. (Except when especially necessary to show the dependence on pion-mass, we shall choose units $m_{\pi}=1$ ). Using unitarity and validity of dispersion relations with a finite number of subtractions for $-T<t \leq 0$, Jin and Martin [11]) proved twice subtracted fixed- $t$ dispersion relations for $|t|<t_{0}$. From this Lukaszuk and Martin [12] fixed the constant $C$ in the Froissart bound to obtain 


$$
\sigma_{\text {tot }}(s) \leq_{s \rightarrow \infty} \sigma_{\max }(s),
$$

where

$$
\sigma_{\max }(s) \equiv 4 \pi /\left(t_{0}-\epsilon\right)\left[\ln \left(s / s_{0}\right)\right]^{2},
$$

with $\epsilon$ an arbitrarily small positive constant. The FroissartMartin bounds have inspired much work on high-energy theorems (see e.g. $[13,14])$ and on models of high-energy scattering [15]. Further, Martin proved a bound on the total inelastic cross section $\sigma_{\text {inel }}(s)$ at high energy [16] which is one-fourth of the above bound $\sigma_{\max }(s)$ on the total cross section, and, Wu, Martin, Roy and Singh [17] obtained a bound on $\sigma_{\text {inel }}(s)$ which improves that bound if $\sigma_{\text {tot }}(s)$ is known,

$$
\sigma_{\text {inel }}(s) \leq_{s \rightarrow \infty} \sigma_{\text {tot }}(s)\left(1-\sigma_{\text {tot }}(s) / \sigma_{\max }(s)\right) .
$$

The motivation for getting a bound on the inelastic cross section is the almost general belief [15] that at high energies hadron total cross sections cannot exceed twice the inelastic cross section. Hence, gaining a factor of 4 in the inelastic cross section gains a factor of 2 in the total cross section.

One shortcoming of these bounds from the standpoint of rigor is that [18] they are deduced by assuming that the absorptive part $A(s, t), 0 \leq t<t_{0}$ is bounded by Const $s^{2} / \ln \left(s / s_{0}\right)$ for $s \rightarrow \infty$, whereas axiomatic field theory results only guarantee that

$$
C(t) \equiv \int_{s_{\mathrm{th}}}^{\infty} d s A(s, t) / s^{3}<\infty, \quad 0 \leq t<t_{0},
$$

where $s_{\text {th }}$ is the $s$-channel threshold. From the practical point of view, a more serious shortcoming is that they involve the unknown scale factor $s_{0}$ in the argument of the logarithm and the unknown arbitrarily small but nonzero constant $\epsilon$.

In the case of the total cross section, both these shortcomings were removed recently [1]. Bounds on energy averages of the total cross section were obtained in which the scale $s_{0}$ is determined in terms of $C(t)$. $C(t)$ can be bounded rigorously in terms of pion mass alone for $\pi^{0} \pi^{0}$ scattering. Thus we obtained the absolute bound [1],

$$
\begin{aligned}
\bar{\sigma}_{\text {tot }}(s, \infty) \leq & \pi\left(m_{\pi}\right)^{-2}\left[\ln \left(s / s_{0}\right)+(1 / 2) \ln \ln \left(s / s_{0}\right)+1\right]^{2} \\
& +O\left(\ln \ln \left(s / s_{0}\right)\right), \\
s_{0}^{-1}= & 17 \pi \sqrt{\pi / 2} m_{\pi}^{-2},
\end{aligned}
$$

where,

$$
\bar{\sigma}_{\text {tot }}(s, \infty) \equiv s \int_{s}^{\infty} d s^{\prime} \sigma_{\text {tot }}\left(s^{\prime}\right) / s^{\prime 2} .
$$

We also obtained somewhat improved bounds by using the additional phenomenological inputs for the $D$-wave scattering length [19] for pion-pion scattering.
We prove here analogous bounds on energy averages of the inelastic cross section. We choose the same normalizations as in Martin-Roy [1]. $F(s, t)$ denotes an $a b \rightarrow a b$ scattering amplitude at c.m. energy $\sqrt{s}$ and momentum transfer squared $t$ normalized for nonidentical partcles $a, b$ such that the differential cross section $d \sigma / d \Omega(s, t)$ is given by $|4 F(s, t) / \sqrt{s}|^{2}$, with $t$ being given in terms of the c.m. momentum $k$ and the scattering angle $\theta$ by the relation,

$$
t=-2 k^{2}(1-\cos \theta) ; \quad z \equiv \cos \theta=1+t /\left(2 k^{2}\right) .
$$

Then, for fixed $s$ larger than the physical $s$-channel threshold, $F(s ; \cos \theta) \equiv F(s, t)$ is analytic in the complex $\cos \theta$-plane inside the Lehmann-Martin ellipse with foci -1 and +1 and semi-major axis $\cos \theta_{0}=1+t_{0} /\left(2 k^{2}\right)$. Within the ellipse, in particular, for $|t|<t_{0}, F(s, t)$ has the convergent partial wave expansion,

$$
F(s, t)=\frac{\sqrt{s}}{4 k} \sum_{l=0}^{\infty}(2 l+1) P_{l}(z) a_{l}(s),
$$

with the unitarity constraint,

$$
\operatorname{Im} a_{l}(s) \geq\left|a_{l}(s)\right|^{2}, \quad s \geq s_{\mathrm{th}} .
$$

Correspondingly, the optical theorem gives, for nonidentical partcles $a, b$,

$$
\begin{aligned}
\sigma_{\text {tot }}(s) & =\frac{4 \pi}{k} \operatorname{Im}(4 F(s, 0) / \sqrt{s}) \\
& =\frac{4 \pi}{k^{2}} \sum_{l=0}^{\infty}(2 l+1) \operatorname{Im} a_{l}(s) .
\end{aligned}
$$

For identical particles, e.g. for $\pi^{0} \pi^{0}$ scattering, or for pion-pion scattering with isospin $I$, the partial waves $a_{l}(s) \rightarrow 2 a_{l}^{I}(s)$ in the partial wave expansion, i.e.

$$
\begin{gathered}
F^{I}(s, t)=\frac{\sqrt{s}}{4 k} \sum_{l=0}^{\infty}(2 l+1) 2 a_{l}^{I}(s) P_{l}(z), \\
\sigma_{\mathrm{tot}}^{I}(s)=\frac{4 \pi}{k^{2}} \sum_{l=0}^{\infty}(2 l+1) 2 \operatorname{Im} a_{l}^{I}(s),
\end{gathered}
$$

and we have the same formula for the differential cross section in terms of $F(s, t)$, and the same form of the unitarity constraint, $\operatorname{Im} a_{l}^{I}(s) \geq\left|a_{l}^{I}(s)\right|^{2}, s \geq 4$, as for nonidentical particles. At threshold, $F^{I}(4,0)=a_{0}^{I}$, the S-wave scattering length for isospin $I$.

It will be seen that proofs of the bounds for inelastic cross sections are considerably more involved than those for total cross sections, but the basic principles are the same. We give detailed derivations for the case of 
nonidentical particles $a \neq b$ and also quote the identical particle results when needed.

\section{CONVEXITY PROPERTIES OF LOWER BOUND ON ABSORPTIVE PART IN TERMS OF TOTAL INELASTIC CROSS SECTION}

We obtain a lower bound on the absorptive part of $F(s, t)$ (for $s \geq s_{\text {th }}$ and $0 \leq t<t_{0}$ ) in terms of the inelastic cross section $\sigma_{\text {inel }}(s)$. Following [20] we prove that the bound is a convex function of the inelastic cross section. The absorptive part has the partial wave expansion,

$$
F_{s}(s, t) \equiv A(s, t)=\frac{\sqrt{s}}{4 k} \sum_{l=0}^{\infty}(2 l+1) P_{l}(z) \operatorname{Im} a_{l}(s) .
$$

The corresponding expansion of the inelastic cross section is

$$
\sigma_{\text {inel }}(s)=\frac{4 \pi}{k^{2}} \sum_{l=0}^{\infty}(2 l+1)\left(\operatorname{Im} a_{l}(s)-\left|a_{l}(s)\right|^{2}\right) .
$$

Actually we shall vary $\operatorname{Im} a_{l}(s)$ subject to the positivity restrictions (due to unitarity),

$$
\operatorname{Im} a_{l}(s) \geq 0,
$$

to minimize $A(s, t)$, given

$$
\sigma_{\text {inel, }, \mathrm{im}}(s) \equiv \frac{4 \pi}{k^{2}} \sum_{l=0}^{\infty}(2 l+1)\left(\operatorname{Im} a_{l}(s)-\left(\operatorname{Im} a_{l}(s)\right)^{2}\right) .
$$

The bound will be seen to be an increasing function of $\sigma_{\text {inel,im }}(s)$. Further,

$$
\sigma_{\text {inel,im }}(s) \geq \sigma_{\text {inel }}(s)
$$

therefore, the bound will still hold when we replace $\sigma_{\text {inel,im }}(s)$ by the experimentally accessible $\sigma_{\text {inel }}(s)$. We work at a fixed $s$; so, unless specially needed, we shall suppress the $s$ dependence of $\operatorname{Im} a_{l}(s), \sigma_{\text {inel,im }}(s)$ and $\sigma_{\text {inel }}(s)$. The Lagrange multiplier method with positivity constraints on partial waves gives the variational solution $\left(\operatorname{Im} a_{l}\right)_{0}$,

$$
\begin{aligned}
& \left(\operatorname{Im} a_{l}\right)_{0}=\frac{1}{2}\left(1-\frac{P_{l}(z)}{P_{\lambda}(z)}\right), \quad l \leq L ; L \leq \lambda<L+1 \\
& \left(\operatorname{Im} a_{l}\right)_{0}=0, \quad l>L,
\end{aligned}
$$

where the integer $L$ and the non-negative fraction $\lambda-L$ are to be determined so as to reproduce the given $\sigma_{\text {inel,im; }}$, here $P_{\lambda}(z)$ for noninteger $\lambda$ and $z \geq 1$ is defined by

$$
P_{\lambda}(z)=\frac{1}{\pi} \int_{0}^{\pi}\left(z+\cos \phi \sqrt{z^{2}-1}\right)^{\lambda} d \phi .
$$

If $A_{0}(s, t)$ denotes the absorptive part with partial waves $\left(\operatorname{Im} a_{l}\right)_{0}$ and $A(s, t)$ that with arbitrary positive partial waves with the given $\sigma_{\text {inel,im }}(s)$, we obtain by direct subtraction,

$$
\begin{aligned}
& 4(k / \sqrt{s})\left(A(s, t)-A_{0}(s, t)\right) \\
& =P_{\lambda}(z) \sum_{l=0}^{L}(2 l+1)\left(\operatorname{Im} a_{l}-\left(\operatorname{Im} a_{l}\right)_{0}\right)^{2}+ \\
& \quad \times P_{\lambda}(z) \sum_{l=L+1}^{\infty}(2 l+1)\left(\left(\operatorname{Im} a_{l}\right)^{2}+\operatorname{Im} a_{l}\left(\frac{P_{l}(z)}{P_{\lambda}(z)}-1\right)\right) \\
& \geq 0 .
\end{aligned}
$$

The last inequality follows because for $z \geq 1$, and $\lambda \geq 0$, $P_{\lambda}(z)$ is an increasing function of $\lambda$. We then have

$$
\begin{aligned}
4(k / \sqrt{s}) A(s, t) & \geq \sum_{l=0}^{L}(2 l+1) P_{l}(z) \frac{1}{2}\left(1-\frac{P_{l}(z)}{P_{\lambda}(z)}\right) \\
& \equiv A(\lambda),
\end{aligned}
$$

where

$$
\begin{aligned}
\sigma_{\text {inel.im }} \frac{k^{2}}{4 \pi} & =\sum_{l=0}^{l=L}(2 l+1)(1 / 4)\left(1-\left(\frac{P_{l}(z)}{P_{\lambda}(z)}\right)^{2}\right) \\
& \equiv \Sigma_{I}(\lambda) .
\end{aligned}
$$

Note that $\Sigma_{I}(\lambda)$ and $A(\lambda)$ are monotonically increasing continuous functions of $\lambda$; hence, $\lambda$ and $A(\lambda)$ may be considered functions of $\Sigma_{I}$, and

$$
d A / d \Sigma_{I}=\left((d A / d \lambda) /\left(d \Sigma_{I} / d \lambda\right)\right)=P_{\lambda}(z),
$$

which is always positive and also continuous at integer $\lambda$, although $(d A / d \lambda)$ and $\left(d \Sigma_{I} / d \lambda\right)$ are discontinuous there. Hence,

$$
d^{2} A / d \Sigma_{I}^{2}=\left(\left(d P_{\lambda}(z) / d \lambda\right) /\left(d \Sigma_{I} / d \lambda\right)\right)>0,
$$

which is discontinuous at integer $\lambda$, but always positive. This completes the proof that $A\left(\lambda\left(\Sigma_{I}\right)\right)$ is a convex function of $\Sigma_{I}$; i.e. at a given $s$ the lower bound on $A(s, t)$ is an increasing and convex fuction of $\sigma_{\text {inel.im }}$ and, hence, of $\sigma_{\text {inel }}$.

\section{EXPLICIT EVALUATION OF THE BOUND}

Explicitly,

$$
A\left(\lambda\left(\Sigma_{I}\right)\right)=\int_{0}^{\Sigma_{I}} \frac{d A}{d \Sigma_{I}^{\prime}} d \Sigma_{I}^{\prime}=\int_{0}^{\Sigma_{I}} P_{\lambda^{\prime}}(z) d \Sigma_{I}^{\prime},
$$


where $\lambda^{\prime}, L^{\prime}$ corresponds to the value $\Sigma_{I}^{\prime}$ of $\sigma_{\text {inel.im }} k^{2} /(4 \pi)$. When $0<\Sigma_{I}^{\prime}<\left(1-z^{-2}\right) / 4 \equiv \Sigma_{I}(1)$ we get $L^{\prime}=0$ and the corresponding part of the integral can be evaluated exactly. Hence,

$$
A\left(\lambda\left(\Sigma_{I}\right)\right)=\left(1-z^{-1}\right) / 2+\int_{\Sigma_{I}(1)}^{\Sigma_{I}} P_{\lambda^{\prime}}(z) d \Sigma_{I}^{\prime} .
$$

In the remaining integral $L^{\prime} \geq 1$ and we shall prove that for $\Sigma_{I}^{\prime} \geq \Sigma_{I}(1)$

$$
\left(\lambda^{\prime}\right)^{2} \geq 4 \Sigma_{I}^{\prime}
$$

Proof--From the partial wave expansion for $\Sigma_{I}\left(\lambda^{\prime}\right) \equiv \Sigma_{I}^{\prime}$, we obtain

$$
4 \Sigma_{I}^{\prime} \leq\left(L^{\prime}\right)^{2}+\left(2 L^{\prime}+1\right)\left(1-\left(\frac{P_{L^{\prime}}(z)}{P_{\lambda^{\prime}}(z)}\right)^{2}\right) .
$$

The integral representation for $P_{\lambda}(z)$ given before yields, for $z>1$,

$$
\begin{aligned}
\left(\frac{P_{L^{\prime}}(z)}{P_{\lambda^{\prime}}(z)}\right)^{2} & \geq \exp \left(-2\left(\lambda^{\prime}-L^{\prime}\right) \ln \left(z+\sqrt{z^{2}-1}\right)\right) \\
& \geq 1-2\left(\lambda^{\prime}-L^{\prime}\right) \ln \left(z+\sqrt{z^{2}-1}\right)
\end{aligned}
$$

where the last inequality uses $\exp (-x) \geq 1-x$. At high energies $\ln \left(z+\sqrt{z^{2}-1}\right)$ goes to zero, and we assume moderately high energies $\left(k>6 m_{\pi}\right)$ such that, with $t<4 m_{\pi}^{2}$,

$$
\ln \left(z+\sqrt{z^{2}-1}\right)<1 / 3 .
$$

Then,

$$
\begin{aligned}
4 \Sigma_{I}^{\prime} & \leq\left(L^{\prime}\right)^{2}+\left(2 L^{\prime}+1\right) 2\left(\lambda^{\prime}-L^{\prime}\right) \ln \left(z+\sqrt{z^{2}-1}\right) \\
& \leq\left(\lambda^{\prime}\right)^{2}-\left(\lambda^{\prime}-L^{\prime}\right)\left(\lambda^{\prime}+L^{\prime}-(2 / 3)\left(\left(2 L^{\prime}+1\right)\right)\right) \\
& \leq\left(\lambda^{\prime}\right)^{2}, \quad \text { for } L^{\prime} \geq 1,
\end{aligned}
$$

which completes the proof.

Since $P_{\lambda^{\prime}}(z)$ is an increasing function of $\lambda^{\prime}$, we obtain,

$$
A\left(\lambda\left(\Sigma_{I}\right)\right)=\left(1-z^{-1}\right) / 2+\int_{\Sigma_{I}(1)}^{\Sigma_{I}} P \sqrt{4 \Sigma_{I}^{\prime}}(z) d \Sigma_{I}^{\prime} .
$$

Using the integral representation for $P_{\lambda}(z)$ given before and the analogous representation

$$
I_{0}(z)=\frac{1}{\pi} \int_{0}^{\pi} \exp (z \cos \phi) d \phi
$$

for the modified Bessel function, and the elementary inequality

$$
\begin{aligned}
& \ln \left(\left(z+\cos \phi \sqrt{z^{2}-1}\right) \geq(\cos \phi) \ln \left(\left(z+\sqrt{z^{2}-1}\right),\right.\right. \\
& z>1,
\end{aligned}
$$

we obtain [20]

$$
\begin{aligned}
& P_{\lambda}(z) \geq I_{0}\left(\lambda \ln \left(\left(z+\sqrt{z^{2}-1}\right)\right)\right. \\
& \geq I_{0}\left(\lambda \sqrt{z^{2}-1} / z\right), \quad z>1 .
\end{aligned}
$$

Substituting the above inequalities, the integral over $\Sigma_{I}^{\prime}$ in the expression for the lower bound can be evaluated exactly, and we have the exact result (without any highenergy approximation),

$$
\begin{gathered}
4(k / \sqrt{s}) A(s, t) \geq A\left(\Sigma_{I}\right)>\frac{\left(1-z^{-1}\right)}{2} \\
+\left.(1 / 2)\left(\ln \left(\left(z+\sqrt{z^{2}-1}\right)\right)\right)^{-2}\left[x^{\prime} I_{1}\left(x^{\prime}\right)\right]\right|_{x^{\prime}=u_{1}} ^{x^{\prime}=u}, \\
u \equiv \ln \left(\left(z+\sqrt{z^{2}-1}\right)\right) \sqrt{k^{2} \sigma_{\text {inel }} / \pi} \\
u_{1} \equiv \ln \left(\left(z+\sqrt{z^{2}-1}\right)\right) \sqrt{1-z^{-2}},
\end{gathered}
$$

and the slightly weaker but simpler result,

$$
\begin{aligned}
A\left(\Sigma_{I}\right)> & \frac{z^{2}}{2\left(z^{2}-1\right)} \frac{\sqrt{4 \Sigma_{I}\left(z^{2}-1\right)}}{z} I_{1}\left(\frac{\sqrt{4 \Sigma_{I}\left(z^{2}-1\right)}}{z}\right) \\
& +\frac{\left(1-z^{-1}\right)}{2}-\frac{1}{2} I_{1}\left(\frac{z^{2}-1}{z^{2}}\right) .
\end{aligned}
$$

Note that at high energies $z-1 \rightarrow 0$, and the last two terms give only a small positive contribution,

$$
\frac{\left(1-z^{-1}\right)}{2}-\frac{1}{2} I_{1}\left(\frac{z^{2}-1}{z^{2}}\right) \approx(z-1)^{2} / 4, \quad z-1 \rightarrow 0 .
$$

Hence, at sufficiently high energies, but without any highenergy approximation, we have the bound given by Eqs. (37)-(38) and the slightly weaker but simpler bound,

$$
\begin{aligned}
A(s, t) & >\frac{k \sqrt{s}}{4 t} \frac{z^{2}}{z+1} x I_{1}(x), \\
x & \equiv \sqrt{\frac{z+1}{2 z^{2}}} \sqrt{\frac{t \sigma_{\text {inel }}(s)}{\pi}} .
\end{aligned}
$$

\section{BOUND ON ENERGY-AVERAGED INELASTIC CROSS SECTION}

Multiplying by $s^{-3}$ and integrating over $s$, we obtain a lower bound on $C_{\left(s_{1}, s_{2}\right)}(t)$ which is the contribution from $s_{1}$ to $s_{2}$ to $C(t)$, 


$$
\begin{aligned}
C_{\left(s_{1}, s_{2}\right)}(t) & \equiv \int_{s_{1}}^{s_{2}} A\left(s^{\prime}, t\right) \frac{d s^{\prime}}{\left(s^{\prime}\right)^{3}} \\
& \geq \int_{s_{1}}^{s_{2}} \frac{d s^{\prime}}{\left(s^{\prime}\right)^{2}} \frac{\sqrt{\left(1-4 / s_{1}\right)}}{16 t} x_{1}^{\prime} I_{1}\left(x_{1}^{\prime}\right),
\end{aligned}
$$

where $s_{2}>s_{1}$, and we used $2 k^{\prime} / \sqrt{s^{\prime}}>\sqrt{\left(1-4 / s_{1}\right)}$, and $2 z_{1}^{2} /\left(z_{1}+1\right) \geq 2 z^{2} /(z+1) \geq 1$ for $s^{\prime}$ in the interval $\left(s_{1}, s_{2}\right)$, and

$$
z_{1} \equiv \frac{s_{1}-4+2 t}{s_{1}-4}, \quad x_{1}^{\prime} \equiv \sqrt{\frac{z_{1}+1}{2 z_{1}^{2}}} \sqrt{\frac{t \sigma_{\text {inel }}\left(s^{\prime}\right)}{\pi}} .
$$

The lower bound on $C\left(s_{1}, s_{2}\right)(t)$ is an average with the normalized weight function

$$
\rho\left(s^{\prime}\right)=\frac{s_{1} s_{2}}{\left(s_{2}-s_{1}\right)\left(s^{\prime}\right)^{2}}
$$

of an integrand which is a convex function of $\sigma_{\text {inel }}\left(s^{\prime}\right)$. The convexity is readily proved; using $\left(x I_{1}(x)\right)^{\prime}=x I_{0}(x)$, and denoting

$$
t_{1}=t \frac{z_{1}+1}{2 z_{1}^{2}}=t \frac{\left(s_{1}-4\right)\left(s_{1}-4+t\right)}{\left(s_{1}-4+2 t\right)^{2}}<t
$$

we have

$$
\frac{d\left(x_{1}^{\prime} I_{1}\left(x_{1}^{\prime}\right)\right)}{d \sigma_{\text {inel }}\left(s^{\prime}\right)}=\frac{t_{1}}{2 \pi} I_{0}\left(\sqrt{\frac{t_{1} \sigma_{\text {inel }}\left(s^{\prime}\right)}{\pi}}\right) .
$$

Since the right-hand side is an increasing function of $\sigma_{\text {inel }}\left(s^{\prime}\right)$, we get the convexity property,

$$
\frac{d^{2}\left(x_{1}^{\prime} I_{1}\left(x_{1}^{\prime}\right)\right)}{d \sigma_{\text {inel }}\left(s^{\prime}\right)^{2}}>0 .
$$

Since the average of a convex function is greater than the convex function of the average [21], we have the bound

$$
C_{\left(s_{1}, s_{2}\right)}(t) \geq \frac{s_{2}-s_{1}}{16 t s_{1} s_{2}} \sqrt{\left(1-4 / s_{1}\right)} x_{1} I_{1}\left(x_{1}\right)
$$

where

$$
\begin{gathered}
x_{1} \equiv \sqrt{\frac{t_{1} \bar{\sigma}_{\text {inel }}\left(s_{1}, s_{2}\right)}{\pi}}, \\
\bar{\sigma}_{\text {inel }}\left(s_{1}, s_{2}\right) \equiv \int_{s_{1}}^{s_{2}} d s^{\prime} \rho\left(s^{\prime}\right) \sigma_{\text {inel }}\left(s^{\prime}\right) .
\end{gathered}
$$

To get bounds on $\bar{\sigma}_{\text {inel }}(s, \infty)$, and $\bar{\sigma}_{\text {inel }}(s, 2 s)$, we just choose the corresponding values for $\left(s_{1}, s_{2}\right)$. Thus we obtain, without any asymptotic approximations in $\mathrm{s}$,

$$
\begin{gathered}
C_{(s, \infty)}(t) \geq \frac{1}{16 t s} \sqrt{(1-4 / s)} x_{1} I_{1}\left(x_{1}\right), \\
x_{1} \equiv \sqrt{\frac{t_{1} \bar{\sigma}_{\text {inel }}(s, \infty)}{\pi}},
\end{gathered}
$$

and

$$
\begin{gathered}
C_{(s, 2 s)}(t) \geq \frac{1}{32 t s} \sqrt{(1-4 / s)} x_{2} I_{1}\left(x_{2}\right), \\
x_{2} \equiv \sqrt{\frac{t_{1} \bar{\sigma}_{\text {inel }}(s, 2 s)}{\pi}},
\end{gathered}
$$

where

$$
t_{1}=t \frac{(s-4)(s-4+t)}{(s-4+2 t)^{2}}<t .
$$

\section{A. Asymptotic bounds}

Since we want asymptotic upper bounds on the energyaveraged inelastic cross sections, we can assume without loss of generality that the arguments $x_{1}, x_{2}$ of the modified Bessel functions tend to infinity and obtain

$$
\begin{gathered}
16 s t C_{(s, \infty)}(t) \sqrt{2 \pi}>\left(\sqrt{\xi_{1}} \exp \xi_{1}\right)\left(1+O\left(1 / \xi_{1}\right)\right), \\
\xi_{1}=\sqrt{\frac{t \bar{\sigma}_{\text {inel }}(s, \infty)}{\pi}},
\end{gathered}
$$

and

$$
\begin{gathered}
32 s t C_{(s, 2 s)}(t) \sqrt{2 \pi}>\left(\sqrt{\xi_{2}} \exp \xi_{2}\right)\left(1+O\left(1 / \xi_{2}\right)\right), \\
\xi_{2}=\sqrt{\frac{t \bar{\sigma}_{\text {inel }}(s, 2 s)}{\pi}} .
\end{gathered}
$$

We now use the elementary lemma proved in [20] and [1]. Lemma.-If $\xi>1$, and $y \geq \sqrt{\xi} \exp \xi$, then

$$
\xi<f(y) \equiv \ln y-(1 / 2) \ln \left(\ln y-\frac{1}{2} \ln \ln y\right) .
$$

With $f(y)$ as defined above, we obtain the asymptotic bounds,

$$
\bar{\sigma}_{\text {inel }}(s, \infty) \leq_{s \rightarrow \infty} \frac{\pi}{t}\left(f\left(4 s / s_{0}\right)\right)^{2},
$$

where 


$$
\frac{1}{s_{0}}=4 t C_{(s, \infty)}(t) \sqrt{2 \pi}, \quad t=4 m_{\pi}^{2}-\epsilon,
$$

and

$$
\bar{\sigma}_{\text {inel }}(s, 2 s) \leq_{s \rightarrow \infty} \frac{\pi}{t}\left(f\left(8 s / s_{0}\right)\right)^{2} .
$$

Notice that the coefficients of $(\ln s)^{2}$ in these bounds on the inelastic cross section are one-fourth of those in the corresponding bounds on the total cross section at high energies, and the scale factors in the inelastic case are also one-fourth of those in the corresponding total cross-section bounds [1],

$$
\begin{aligned}
& \bar{\sigma}_{\text {tot }}(s, \infty) \leq_{s \rightarrow \infty} \frac{4 \pi}{t}\left(f\left(s / s_{0}\right)\right)^{2}, \\
& \bar{\sigma}_{\text {tot }}(s, 2 s) \leq_{s \rightarrow \infty} \frac{4 \pi}{t}\left(f\left(2 s / s_{0}\right)\right)^{2} .
\end{aligned}
$$

In the case of pion-pion scattering, we may remove the unknown $\epsilon$ in $t=4 m_{\pi}^{2}-\epsilon$ rigorously by using absolute bounds on the $D$ wave below threshold derived in [1], or use phenological inputs on the $D$-wave scattering length and set $\epsilon=0$. The main qualitative difference from the case of nonidentical particles is that only even partial waves occur in $\pi^{0} \pi^{0}$ scattering, We first show that in spite of this difference, the bounds of this section at moderate energies as well as the asymptotic bounds on inelastic cross sections hold for $\pi^{0} \pi^{0}$ scattering.

\section{BOUNDS ON PION-PION INELASTIC CROSS SECTIONS}

We shall exploit isospin invariance,

$$
\begin{aligned}
F^{\pi^{0} \pi^{0} \rightarrow \pi^{0} \pi^{0}} & =\frac{1}{3} F^{0}+\frac{2}{3} F^{2} \\
& =\frac{\sqrt{s}}{4 k} \sum_{l=0,2}^{\infty}(2 l+1) 2 a_{l}^{\pi^{0} \pi^{0} \rightarrow \pi^{0} \pi^{0}}(s) P_{l}(z), \\
F^{\pi^{0} \pi^{0} \rightarrow \pi^{+} \pi^{-}} & =\frac{1}{3} F^{0}-\frac{1}{3} F^{2} \\
& =\frac{\sqrt{s}}{4 k} \sum_{l=0,2}^{\infty}(2 l+1) \sqrt{2} a_{l}^{\pi^{0} \pi^{0} \rightarrow \pi^{+} \pi^{-}}(s) P_{l}(z) .
\end{aligned}
$$

Unitarity, then, implies

$$
\begin{aligned}
\operatorname{Im} a_{l}^{\pi^{0} \pi^{0} \rightarrow \pi^{0} \pi^{0}} & \geq\left|a_{l}^{\pi^{0} \pi^{0} \rightarrow \pi^{0} \pi^{0}}\right|^{2}+\left|a_{l}^{\pi^{0} \pi^{0} \rightarrow \pi^{+} \pi^{-}}\right|^{2} \\
& =\frac{1}{3}\left|a_{l}^{0}(s)\right|^{2}+\frac{2}{3}\left|a_{l}^{2}(s)\right|^{2} .
\end{aligned}
$$

Hence, we define the inelastic cross section considering $\pi^{0} \pi^{0} \rightarrow \pi^{+} \pi^{-}$also as an elastic channel,

$$
\begin{aligned}
\sigma_{\text {inel }}^{\pi^{0} \pi^{0}} \equiv & \sigma_{\text {tot }}^{\pi^{0} \pi^{0}}-\sigma^{\pi^{0} \pi^{0} \rightarrow \pi^{0} \pi^{0}}-\sigma^{\pi^{0} \pi^{0} \rightarrow \pi^{+} \pi^{-}} \\
= & \frac{8 \pi}{k^{2}} \sum_{l=0,2}^{\infty}(2 l+1) \\
& \times\left(\frac{1}{3}\left(\operatorname{Im} a_{l}^{0}-\left|a_{l}^{0}\right|^{2}\right)+\frac{2}{3}\left(\operatorname{Im} a_{l}^{2}-\left|a_{l}^{2}\right|^{2}\right)\right) .
\end{aligned}
$$

Note that

$$
\begin{aligned}
\sigma_{\text {inel }}^{\pi^{0} \pi^{0}} & \leq \sigma_{\text {inel,im }}^{\pi^{0} \pi^{0}} \\
\equiv & \frac{8 \pi}{k^{2}} \sum_{l=0,2,}^{\infty}(2 l+1) \\
& \times\left(\frac{1}{3}\left(\operatorname{Im} a_{l}^{0}-\left(\operatorname{Im} a_{l}^{0}\right)^{2}\right)+\frac{2}{3}\left(\operatorname{Im} a_{l}^{2}-\left(\operatorname{Im} a_{l}^{2}\right)^{2}\right)\right) .
\end{aligned}
$$

As before, we vary the $\operatorname{Im} a_{l}^{I}$ subject to positivity constraints and the given $\sigma_{\text {inel,im }}^{\pi^{0} \pi^{0}}$ to minimize the absorptive part,

$$
\begin{aligned}
& A^{\pi^{0} \pi^{0} \rightarrow \pi^{0} \pi^{0}}(s, t) \\
& =\frac{\sqrt{s}}{4 k} \sum_{l=0,2}^{\infty}(2 l+1) \\
& \times P_{l}(z) 2\left(\frac{1}{3}\left(\operatorname{Im} a_{l}^{0}\right)+\frac{2}{3}\left(\operatorname{Im} a_{l}^{2}\right)\right) .
\end{aligned}
$$

The minimum is reached when

$\operatorname{Im} a_{l}^{0}=\operatorname{Im} a_{l}^{2}=\frac{1}{2}\left(1-\frac{P_{l}(z)}{P_{\lambda}(z)}\right), \quad l \leq L ;$

$\operatorname{Im} a_{l}^{0}=\operatorname{Im} a_{l}^{2}=0, \quad l>L, \quad L \leq \lambda<L+2$.

The minimum is an increasing and convex function of $\sigma_{\text {inel,im }}^{\pi^{0} \pi^{0}}$. The lower bound on the absorptive part, therefore, remains valid if we replace $\sigma_{\text {inel,im }}^{\pi^{0} \pi^{0}}$ by $\sigma_{\text {inel }}^{\pi^{0} \pi^{0}}$. Again, defining

$$
\begin{gathered}
4(k / \sqrt{s}) A^{\pi^{0} \pi^{0} \rightarrow \pi^{0} \pi^{0}}(s, t) \equiv A^{\pi^{0} \pi^{0}}(\lambda), \\
\sigma_{\text {inel }}^{\pi^{0} \pi^{0}} \frac{k^{2}}{4 \pi} \equiv \Sigma_{I}^{\pi^{0} \pi^{0}}(\lambda),
\end{gathered}
$$

we prove that if $\ln \left(z+\sqrt{z^{2}-1}\right)<1 / 6$, which holds at moderately high energies, $\Sigma_{I}^{\pi^{0} \pi^{0}}(\lambda)<\lambda^{2} / 4$ if $L \geq 2$. Finally, proceeding as for nonidentical particles, we obtain a bound without any high-energy approximations,

$$
A^{\pi^{0} \pi^{0}}\left(\lambda\left(\Sigma_{I}^{\pi^{0} \pi^{0}}\right)\right) \geq\left(1-1 / P_{2}(z)\right)+\int_{\Sigma_{I}^{\pi^{0} \pi^{0}}(2)}^{\Sigma_{\pi^{0} \pi^{0}}} P \sqrt{4 \Sigma_{I}^{\prime}}(z) d \Sigma_{I}^{\prime} .
$$


This yields the exact bound,

$$
\begin{aligned}
4(k / \sqrt{s}) A^{\pi^{0} \pi^{0}}(s, t) & \\
\left.\geq\left(1-1 / P_{2}(z)\right)+(1 / 2) \alpha(z)^{-2}\left[x^{\prime} I_{1}\left(x^{\prime}\right)\right]\right]_{x^{\prime}=v_{1}}^{x^{\prime}=v}, & \\
\alpha(z) & \equiv \ln \left(\left(z+\sqrt{z^{2}-1}\right)\right), \\
v & \equiv \alpha(z) \sqrt{\frac{k^{2}}{\pi} \sigma_{\text {inel }}^{\pi^{0} \pi^{0}}} ; \\
v_{1} & \equiv \alpha(z) \sqrt{2\left(1-P_{2}(z)^{-2}\right)} ;
\end{aligned}
$$

a slightly weaker bound is obtained by replacing $\alpha(z)$ by the smaller quantity $\sqrt{z^{2}-1} / z$, and noting that $\left(1-1 / P_{2}(z)\right)-\left.(1 / 2) \alpha(z)^{-2}\left[x^{\prime} I_{1}\left(x^{\prime}\right)\right]\right|_{x^{\prime}=v_{1}}$ is then positive at moderately high energies. Thus, we obtain a slightly weaker but rigorous bound,

$$
\begin{aligned}
A^{\pi^{0} \pi^{0}}(s, t) & >\frac{k \sqrt{s}}{4 t} \frac{z^{2}}{z+1} x I_{1}(x), \\
x & \equiv \sqrt{\frac{z+1}{2 z^{2}}} \sqrt{\frac{t \sigma_{\text {inel }}^{\pi^{0} \pi^{0}}(s)}{\pi}},
\end{aligned}
$$

which is identical to the result given earlier for nonidentical particles. Therefore, the asymptotic bounds of the last section on energy averages of inelastic cross sections also hold for $\pi^{0} \pi^{0}$ scattering.

\section{ABSOLUTE BOUNDS ON $\pi^{0} \pi^{0}$ INELASTIC CROSS SECTIONS}

In [1] we derived absolute bounds on $\pi^{0} \pi^{0} D$ waves below threshold and on $C(t)$ in terms of pion mass alone for $0<t<4$. In particular,

$$
f_{2}(t)<_{t \rightarrow 4-} \frac{4-t}{120}\left(34+6.25(4-t)+O(4-t)^{2}\right),
$$

and

$$
C^{\pi^{0} \pi^{0} \rightarrow \pi^{0} \pi^{0}}(t)<_{t \rightarrow 4-} \frac{17 \pi}{4(4-t)},
$$

where $f_{l}(s)=\frac{\sqrt{s}}{4 k} 2 a_{l}^{\pi^{0} \pi^{0} \rightarrow \pi^{0} \pi^{0}}(s)$. We now use these rigorous bounds in conjunction with the asymptotic bounds on inelastic $\pi^{0} \pi^{0}$ cross sections to remove the unknown $\epsilon=$ $4-t$ in these bounds. The price to pay for the rigor is that we cannot choose $t=4$. For the upper bound on $\bar{\sigma}_{\text {inel }}^{\pi^{0} \pi^{0}}(s, \infty)$, the optimum choice is

$$
\epsilon=4-t=\frac{8}{\ln \left(s / s_{1}\right)},
$$

which yields

$$
\begin{aligned}
& \bar{\sigma}_{\text {inel }}^{\pi^{0} \pi^{0}}(s, \infty) \\
& \quad \leq_{s \rightarrow \infty}(\pi / 4)\left(m_{\pi}\right)^{-2} \times\left(\ln \left(s / s_{1}\right)+(1 / 2) \ln \ln \left(s / s_{1}\right)+1\right)^{2} \\
& \quad+O\left(\ln \ln \left(s / s_{1}\right)\right),
\end{aligned}
$$

where the scale factor $s_{1}$ is given by

$$
1 / s_{1}=34 \pi \sqrt{2 \pi} m_{\pi}^{-2}
$$

and is one-fourth of that for the total cross section case [1]. For the upper bound on $\bar{\sigma}_{\text {inel }}^{\pi^{0} \pi^{0}}(s, 2 s)$, the optimum choice is

$$
\epsilon=4-t=\frac{8}{\ln \left(s / s_{2}\right)},
$$

which yields

$$
\begin{aligned}
\bar{\sigma}_{\text {inel }}^{\pi^{0} \pi^{0}}(s, 2 s) \leq_{s \rightarrow \infty} & (\pi / 4)\left(m_{\pi}\right)^{-2} \\
& \times\left(\ln \left(s / s_{2}\right)+(1 / 2) \ln \ln \left(s / s_{2}\right)+1\right)^{2} \\
& +O\left(\ln \ln \left(s / s_{2}\right)\right),
\end{aligned}
$$

where the scale factor $s_{2}$ is given by

$$
1 / s_{2}=2 / s_{1}=68 \pi \sqrt{2 \pi} m_{\pi}^{-2} .
$$

These are bounds from first principles on a cross section fundamental in strong interaction physics. But for phenomenological comparisons, it is more useful to use some crossed channel low-energy data to get stronger bounds, particularly on the scale of the logarithm.

\section{PHENOMENOLOGICAL COMPARISONS FOR PION-PION SCATTERING}

(i) First, the basic lower bound (from unitarity alone) on the absorptive part $A(s, t)$ in terms of the inelastic cross section, given by Eq. (41), or in terms of the total cross section (Eq. (21) of [1]) can be compared directly with phenomenological estimates of the absorptive part at energies where such estimates are available [22,23]. This can be done for the amplitudes

$$
\begin{aligned}
F^{\pi^{+} \pi^{0} \rightarrow \pi^{+} \pi^{0}(s, t)} & =1 / 2\left(F^{1}+F^{2}\right)(s, t), \\
F^{\pi^{0} \pi^{0} \rightarrow \pi^{0} \pi^{0}}(s, t) & =\frac{1}{3} F^{0}+\frac{2}{3} F^{2}(s, t),
\end{aligned}
$$

which have positive absorptive parts for $s \geq 4,0<t<4$. A violation of the bounds will indicate that the input absorptive part violates unitarity.

(ii) Second, bounds on energy averages of cross sections in the intervals $(s . \infty)$ and $(s .2 s)$ in terms of phenomenological inputs for c.m. energies less than $\sqrt{s}$ follow from unitarity, analyticity and crossing. The crossing relations, 


$$
\begin{aligned}
\frac{1}{2}\left(F^{1}+F^{2}\right)(s, t) & =\frac{1}{3}\left(F^{0}-F^{2}\right)(t, s), \\
F^{\pi^{0} \pi^{0} \rightarrow \pi^{0} \pi^{0}}(s, t) & =F^{\pi^{0} \pi^{0} \rightarrow \pi^{0} \pi^{0}}(t, s),
\end{aligned}
$$

and the Froissart Gribov formula yield

$$
\begin{aligned}
& C_{(s . \infty)}^{\pi^{+} \pi^{0} \rightarrow \pi^{+} \pi^{0}}(t=4) \\
& \quad=\frac{5 \pi}{16} m_{\pi}\left(a_{2}^{0}-a_{2}^{2}\right)-C_{(4, s)}^{\pi^{+} \pi^{0} \rightarrow \pi^{+} \pi^{0}}(t=4)
\end{aligned}
$$

and

$$
\begin{aligned}
& C_{(s, \infty)}^{\pi^{0} \pi^{0} \rightarrow \pi^{0} \pi^{0}}(t=4) \\
& \quad=\frac{5 \pi}{16} m_{\pi}\left(a_{2}^{0}+2 a_{2}^{2}\right)-C_{(4, s)}^{\pi^{0} \pi^{0} \rightarrow \pi^{0} \pi^{0}}(t=4) .
\end{aligned}
$$

Here, as in [1], we defined the $l$-wave scattering lengths $a_{l}^{I}$ as the $q \rightarrow 0$ limits of the phase shifts $\delta_{l}^{I}(q)$ divided by $q^{2 l+1}$ where $q$ is the c.m. momentum. The Bern group [19] already has estimates of the $D$-wave scattering lengths, and has recently obtained [24] estimates of the absorptive part integrals up to $\sqrt{s}=1,6 \mathrm{GeV}$,

$$
\begin{aligned}
C_{\left(4 m_{\pi}^{2}, s\right)}^{\pi^{+} \pi^{0} \rightarrow \pi^{+} \pi^{0}}(t=4) & =1.48 \times 10^{-3}, C_{\left(4 m_{\pi}^{2}, s\right)}^{\pi^{0} \pi^{0} \rightarrow \pi^{0} \pi^{0}}(t=4) \\
& =2.031 \times 10^{-3} .
\end{aligned}
$$

Hence, the bounds on energy-averaged cross sections $\bar{\sigma}_{\text {inel }}^{\pi^{0} \pi^{0}}(s, 2 s)$ and $\bar{\sigma}_{\text {inel }}^{\pi^{+} \pi^{0}}(s, 2 s)$, as well as $\bar{\sigma}_{\text {inel }}^{\pi^{0} \pi^{0}}(s, \infty)$ and $\bar{\sigma}_{\text {inel }}^{\pi^{+} \pi^{0}}(s, \infty)$ implied by Eqs. (51)-(55) can be directly tested against the corresponding experimental values.

(iii) Third, explicit asymptotic bounds on the averages of the inelastic cross section in the intervals $(s, \infty)$ and $(s, 2 s)$ are given by Eqs. (61) and (63), and in terms of the corresponding averages of the total cross section, in terms of a scale parameter $s_{0} ; s_{0}$ is given by Eq. (62) in terms of $C_{(s, \infty)}$, an integral over absorptive parts in the interval $(s, \infty)$. Substituting the values of the $D$-wave scattering lengths given by [19],

$$
a_{2}^{0} \approx 0.00175 m_{\pi}^{-5} ; \quad a_{2}^{2} \approx 0.00017 m_{\pi}^{-5},
$$

we have, choosing for $s$ a value up to which absorptive parts can be reliably estimated,

$\pi^{0} \pi^{0}: s_{0}^{-1}=m_{\pi}^{-2} 16 \sqrt{2 \pi}\left(2.05 \times 10^{-3}-C_{(4, s)}^{\pi^{0} \pi^{0} \rightarrow \pi^{0} \pi^{0}}(t=4)\right)$,

$\pi^{+} \pi^{0}: s_{0}^{-1}=m_{\pi}^{-2} 16 \sqrt{2 \pi}\left(1.55 \times 10^{-3}-C_{(4, s)}^{\pi^{+} \pi^{0} \rightarrow \pi^{+} \pi^{0}}(t=4)\right)$.
These equations give much stronger bounds than the absolute bounds, e.g., using only positivity of $C_{(4, s)}^{\pi^{0} \pi^{0} \rightarrow \pi^{0} \pi^{0}}(t=4)$, we get

$$
s_{0} \geq 12 m_{\pi}^{2},
$$

which is 800 times the absolute bound $s_{0} \geq .015 m_{\pi}^{2}$. As the absorptive part integrals in the $D$-wave scattering length sum rules are rapidly convergent, even for the moderate value of $\sqrt{s}=1,6 \mathrm{GeV}$, Colangelo et al. [24] obtained a further big improvement in the values of the scale factor when phenomenological values of absorptive parts up to $\sqrt{s}=1,6 \mathrm{GeV}$ are utilized,

$$
\pi^{0} \pi^{0}: s_{0} \geq 1312 m_{\pi}^{2} \quad \pi^{+} \pi^{0}: s_{0} \geq 356 m_{\pi}^{2},
$$

which are not very far from the scale factors used in phenomenological fits [23]. We should remember that the phenomenological values may be dependent on the particular parametrization used to fit experimental cross sections. The implicit bounds (51)-(55) discussed in (i) and (ii) are without asymptotic approximations and, therefore, can be compared directly with experiment.

\section{CONCLUDING REMARKS}

In this paper on inelastic cross sections and the previous one on total cross sections [1], we believe we have put the Froissart bound on solid ground by using the notion of average cross sections which avoids completely the problem of the scale in the Froissart bound. These averages can be chosen rather arbitrarily, but once you have chosen one you must stick to it. The simplest averages that we use are the ones from $s$ to infinity and from $s$ to $2 s$. The averaging interval must be sufficiently large if one wants to preserve the coefficients appearing in the Lukaszuk-Martin bound. The only unknown is the value of a certain integral on the absorptive part for some positive $t$. In the special case of pion-pion scattering, all unknown constants are eliminated. The advantage of introducing the bound on the inelastic cross section is that, asymptotically, it is 4 times smaller than the one on the total cross section. So if you accept that the elastic cross section cannot be larger than the inelastic cross section, the limiting case being an expanding black disk, you gain a factor 2 on the bound on the total cross section. However, not everybody agrees with this; for instance, Troshin and Tyurin [25] believe that at high energy the scattering amplitude is dominantly elastic. It is tempting to make a rather daring and nonrigorous suggestion: if the amplitude is essentially elastic (a small inelastic part is unavoidable according to well-known theorems), then the effective large Lehmann ellipse has a right extremity at $t=16 m_{\pi}^{2}$, and the Froissart bound is divided by 4 . 
In any case, a factor of 2 or 4 is not sufficient to bring the absolute bounds near the experimental values [26-28] including the most recent experiments at LHC [29], which indicate a definite increase of the cross sections compatible with a $(\ln (s))^{2}$ behavior. There is little doubt that this trend will continue when LHC reaches higher energies. Towards quantitative improvement, we may find unitarity bounds on the energy averages of the inelastic cross section given the total cross section as an input, in addition to absorptive part integrals at positive $t[30]$.

However, as explained in Sec. VII above, if we are prepared to make phenomenological inputs such as the $D$-wave scattering lengths and low-energy absorptive parts, the situation with respect to experimental comparisons improves dramatically [23].

What can we do on the theoretical side? In the case of pionpion scattering, Kupsch [31] has constructed an amplitude, crossing-symmetric satisfying "inelastic" unitarity and saturating the Froissart bound [31], but he does not give numbers. The result of Gribov [32] shows the importance of satisfying elastic unitarity in the "elastic strips" [33]. This might help, but we do not know how, and there is the problem of finding people interested in working on this.

\section{ACKNOWLEDGMENTS}

We acknowledge very stimulating correspondence with I. Caprini, G. Colangelo, J. Gasser and H. Leutwyler of the University of Bern and thank them for sending us their unpublished results, Eq. (90), on the phenomenological evaluation of the bounds on scale factors for pion-pion scattering. We also thank Maurice Haguenauer for giving us up-to-date references on the work of the Atlas Alpha Collaboration. S. M. R. would like to thank the Indian National Science Academy for an INSA senior scientist grant.
[1] A. Martin and S. M. Roy, Phys. Rev. D 89, 045015 (2014).

[2] M. Froissart, Phys. Rev. 123, 1053 (1961).

[3] A. Martin, Nuovo Cimento 42, 930 (1966).

[4] See e.g. R. F. Streater and A. S. Wightman, PCT. Spin and Statistics and All That (Benjamin, New York, 1978).

[5] H. Epstein, V. Glaser, and A. Martin, Commun. Math. Phys. 13, 257 (1969).

[6] R. Haag and D. Kastler, J. Math. Phys. (N.Y.) 5, 848 (1964); D. Ruelle, Helv. Phys. Acta 35, 147 (1962); H. Araki, Mathematical Theory of Quantum Fields (Oxford University Press, New York, 2000).

[7] Y. I. Azimov, Phys. Rev. D 84, 056012 (2011).

[8] W. Zimmermann, Nuovo Cimento 10, 597 (1958); Nuovo Cimento 21, 249 (1961); 21, 268 (1961).

[9] H. Lehmann, Nuovo Cimento 10, 579 (1958); Fortschr. Phys. 6, 159 (1959).

[10] J. D. Bessis and V. Glaser, Nuovo Cimento 50, 568 (1967); G. Sommer, Nuovo Cimento A A48, 92 (1967); for reviews, see G. Sommer, Fortschr. Phys. 18, 577 (1970); A. Martin, Report No. CERN-TH/99-110, 1999.

[11] Y. S. Jin and A. Martin, Phys. Rev. 135, B1375 (1964).

[12] L. Lukaszuk and A. Martin, Nuovo Cimento A 52A, 122 (1967).

[13] V. Singh and S. M. Roy, Ann. Phys. (N.Y.) 57, 461 (1970).

[14] S. M. Roy, Phys. Rep. 5C, 125 (1972).

[15] H. Cheng and T. T. Wu, Phys. Rev. Lett. 24, 1456 (1970); C. Bourrely, J. Soffer, and T. T. Wu, Phys. Rev. D 19, 3249 (1979) Nucl. Phys. B247, 15 (1984), M. M. Block, Phys. Rep. 436, 71 (2006) and references therein.

[16] A. Martin, Phys. Rev. D 80, 065013 (2009).

[17] T. T. Wu, A. Martin, S. M. Roy, and V. Singh, Phys. Rev. D 84, 025012 (2011).
[18] F. J. Yndurain, Phys. Lett. 31B, 368 (1970); A. K. Common, Nuovo Cimento 69A, 115 (1970).

[19] G. Colangelo, J. Gasser, and H. Leutwyler, Nucl. Phys. B603, 125 (2001); B. Ananthanarayan, G. Colangelo, J. Gasser, and H. Leutwyler, Phys. Rep. 353, 207 (2001).

[20] A. Martin, Pomeranchuk prize lecture, ITEP, Moscow, 2010, https://cds.cern.ch/record/1591290.

[21] G. H. Hardy, J. E. Littlewood, and G. Pólya, Inequalities (Cambridge University Press, Cambridge, England, 1952), p. 74.

[22] I. Caprini, G. Colangelo, J. Gasser, and H. Leutwyler, Phys. Rev. D 68, 074006 (2003); arXiv:hep-ph/0306122.

[23] I. Caprini, G. Colangelo, and H. Leutwyler, Eur. Phys. J. C 72, 1860 (2012); arXiv:1111.7160v2; I. Caprini, G. Colangelo, J. Gasser, and H. Leutwyler (private communication).

[24] I. Caprini, G. Colangelo, and H. Leutwyler (private communication).

[25] S. M. Troshin and N. E. Tyurin, Phys. Lett. 208B, 517 (1988).

[26] U. Amaldi, R. Biancastelli, C. Bosio, G. Matthiae, J. V. Allaby, W. Bartel, G. Cocconi, A. N. Diddens, R. W. Dobinson, and A. M. Wetherell, Phys. Lett. B 44, 112 (1973); S. R. Amendolia et al., Phys. Lett. B 44, 119 (1973).

[27] M. Bozzo et al., Phys. Lett. B 147, 392 (1984); C. Augier et al., Phys. Lett. B 316, 448 (1993).

[28] N. A. Amos et al., Phys. Rev. Lett. 63, 2784 (1989); Phys. Lett. B 243, 158 (1990); Phys. Rev. Lett. 68, 2433 (1992).

[29] Totem Collaboration, Europhys. Lett. 101, 21004 (2013); Atlas-Alpha Collaboration, Nucl. Phys. B889, 486 (2014).

[30] A. Martin and S. M. Roy (to be published). 
[31] J. Kupsch, Nuovo Cimento A 71A, 85 (1982).

[32] V. N. Gribov, Proc. International Conf. on High Energy Physics at Rochester, edited by E. C. G. Sudershan et al. (University of Rochester, Rochester, NY, 1960), p. 340.
[33] A. Martin and J.-M. Richard, Proc. Forward Physics and Luminosity Determination at LHC, Helsinki 2000, edited by K. Haiti et al. (World Scientific, Singapore, 2001), p. 27. 